\title{
Variable Optical Attenuator Based on Long-Range Surface Plasmon Polariton Multimode Interference Coupler
}

\author{
Xiaoqiang Sun, Ying Xie, Tong Liu, Changming Chen, Fei Wang, and Daming Zhang \\ State Key laboratory on Integrated Optoelectronics, Jilin University, Changchun, Jilin 130012, China \\ Correspondence should be addressed to Fei Wang; wang_fei@jlu.edu.cn
}

Received 28 February 2014; Accepted 2 April 2014; Published 22 April 2014

Academic Editor: Tong Zhang

Copyright (C) 2014 Xiaoqiang Sun et al. This is an open access article distributed under the Creative Commons Attribution License, which permits unrestricted use, distribution, and reproduction in any medium, provided the original work is properly cited.

\begin{abstract}
The fabrication and characterization of a thermal variable optical attenuator based on long-range surface plasmon polariton (LRSPP) waveguide with multimode interference architecture were investigated. The surface morphology and waveguide configuration of $\mathrm{Au}$ stripe were studied by atomic force microscopy. The fluctuation of refractive index of poly(methylmethacrylate-glycidyl-methacrylate) polymer cladding was confirmed to be less than $3 \times 10^{-4}$ within $8 \mathrm{~h}$ curing at $120^{\circ} \mathrm{C}$. The end-fire excitation of LRSPP mode guiding at $1550 \mathrm{~nm}$ along Au stripe indicated that the extinction ratio of attenuator was about $12 \mathrm{~dB}$ at a driving power of $69 \mathrm{~mW}$. The measured optical rise time and fall time are 0.57 and $0.87 \mathrm{~ms}$, respectively. These favorable properties promise potentials of this plasmonic device in the application of optical interconnection.
\end{abstract}

\section{Introduction}

Surface plasmon polariton (SPP) is a wave of longitudinal charge oscillations of the conduction electrons at the metal surface. It leads to a transverse magnetic (TM) polarized optical surface waves that propagate along the interface between a metal and a dielectric. SPP has high confinement and sensitivity; however, it decays exponentially into both media with a small penetration depth due to the internal damping in metal, which limits the propagation distance [13]. The attenuation can be significantly reduced by changing a metal-dielectric interface to a symmetrical structure and constructing metal waveguides with finite width and thickness. This will result in the propagation of a lowloss fundamental symmetric mode and an increase in the propagation length, known as the long-range SPP (LRSPP) [4]. The field distribution over the LRSPP waveguide crosssection can extend several micrometers into the cladding, which is close to that of a single mode fiber and facilitates the optical excitation. Research works about the LRSPP waveguide applications for integrated optical components, such as S-bends, Y-junctions, multimode interference (MMI) couplers, and Mach-Zehnder interferometers (MZIs), have been reported [5-10].
Owing to the symmetry request, the exciting of LRSPP is highly sensitive to interface condition, especially the consistence of refractive index between materials on both sides of the metal stripe. Since the optical property of dielectric such as refractive index will change when it is heated due to the thermooptic (TO) effect, the light propagating along LRSPP waveguide will show mode-extinction due to the generated radiation loss. TO modulators, switches, and variable optical attenuators (VOAs) operating in this mode have been realized [10-13]. In these works, the metal stripe both routes the optical signal and acts as the heating element by applying a current through it to affect the effective refractive index of the LRSPP mode via TO effect, which may increase the insertion loss $[14,15]$. This effective modulating of core layer is undoubtedly an advantage of plasmonic devices and favorable for attenuation applications. However, if the thin metal waveguide is used as a heater simultaneously, when the electrical power was applied on the metal stripe, the induced temperature close to $100^{\circ} \mathrm{C}$ may lead to metal stripe deformation that results from the thermal expansion difference between the metal and polymer cladding and worsens when the temperature increases beyond glass transition temperature of polymer $[10,15,16]$. Except for the refractive index variation of the claddings surrounding the metal layer, 


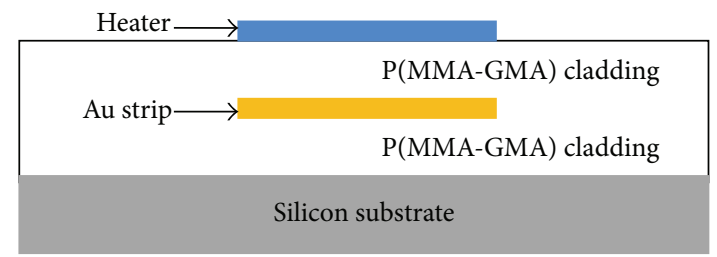

(a)

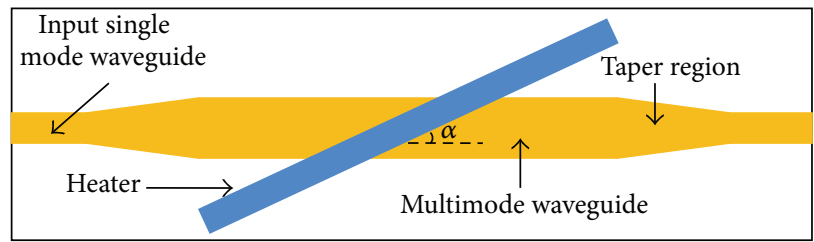

(b)

FIGURE 1: Schematic diagram of (a) cross-sectional view and (b) top view of VOA based on LRSPP waveguide.

the temperature dependent dielectric coefficient of metal may influence the mode characteristics, too [17]. Besides, a visible melting of thin metal films at the electrode may happen due to the mass flow caused by the applied direct current of high densities $\left(>10^{8} \mathrm{~A} / \mathrm{m}^{2}\right)$ [18]. And optically noninvasive electrical contacts make the fabrication process more complex. Thus, in the above configurations, the limited physical lifetime of the thin metal waveguide as a heater is inadequate for commercial applications due to its low thickness of less than $30 \mathrm{~nm}$.

In recent years, various attenuators based on different materials have been investigated. A plasmonic waveguide VOA with a cross-section of $190 \mathrm{~nm} \times 190 \mathrm{~nm}$ shows a low polarization dependent loss of $2.5 \mathrm{~dB}$ [19]. However, due to the direct current applying on metal stripe, its working reliability faces the same problems mentioned above. A compact VOA based on the cutoff effect of photonic crystal waveguide exhibits a variable attenuation range of $29 \mathrm{~dB}$ with a device length of only $16.8 \mu \mathrm{m}$ [20], an electrooptic VOA in silicon-on-insulator achieves a response frequency of about $2 \mathrm{MHz}$ [21], and the monolithic integration of germanium pi-n photodetector with silicon VOA based on submicrometer $\mathrm{Si}$ rib waveguide is demonstrated, too [22]. These works present excellent performance in different aspects. However, techniques of high precise photolithography and etching make the fabrication become very challenging.

Here, we propose a simple VOA structure consisting of a MMI coupler in the form of LRSPP at a wavelength of $1.55 \mu \mathrm{m}$. The index of polymer cladding decreases with its temperature increasing as the upper electrode is heating, so that the effective index of higher-order modes in the waveguide decreases, eventually getting cutoff. The output optical power is then controlled by the generated radiation and mode mismatch to the unheated segments. In this design, the interferential characteristic of MMI waveguide allows a good fabrication tolerance, and the polymer cladding can provide refractive index compatibility with optical fibers, which facilitates optical coupling. Traditional UV photolithography and wet chemical etching techniques imply a low cost fabrication. This proposed that LRSPP based thermal tunable device can be applied in photonic integrated circuit for channel power equalizing in wavelength division multiplex technology [2325].

\section{Thermally Activated LRSPP Attenuator}

2.1. Device Architecture. The proposed LRSPP attenuator architecture supported by a silicon wafer consists of a MMI golden stripe symmetrically sandwiched between polymer claddings. To decrease optical reflection and improve precision, a hyperbolic tapered waveguide with a length of $2050 \mu \mathrm{m}$ is used to connect single mode input/output ports to the MMI section. The coupler is excited with a single $\mathrm{Au}$ stripe from the input side. An aluminum heater is aligned above the metal stripe, as shown in Figure 1. The $30 \mu \mathrm{m}$ thick poly(methyl-methacrylate-glycidyl-methacrylate) (P(MMAGMA)) cladding is on silicon substrate. P(MMA-GMA) material synthesized by ourselves is used in this study due to its stable chemistry properties and relatively low refractive index of about 1.48 at wavelength $1550 \mathrm{~nm}$. The thickness of the Au metal stripe is about $20 \mathrm{~nm}$, which is the compromise of mode confinement and optical propagation loss. The input and output single mode waveguide is designed to have a width of $5 \mu \mathrm{m}$ and a length of $5 \mathrm{~mm}$. The width of MMI waveguide section with centered excitation is $24 \mu \mathrm{m}$. Considering the thermal field distribution across the waveguide and its modulating efficiency, an $8 \mu \mathrm{m}$ wide heater is placed with an optimized angle of $\alpha=1.5^{\circ}$ on top cladding, which is a compromise between the power consumption and attenuation range. When the electrical power is applied to the heater, the generated vertical temperature grades in $\mathrm{P}$ (MMA-GMA) will introduce index change between the upper and lower claddings by TO effect, and then the light under the heater will be reflected at an angle of $2 \alpha$ with respect to the horizontal axis. Supposing that the angle is larger than the fundamental mode of the MMI coupler, the reflected light will be coupled back into higher-order modes that can be filtered out ultimately by the output tapered region and the single mode waveguide. More light will be reflected with the driving power increasing, which results in a larger attenuation [26].

2.2. Multimode Interference Coupler. MMI couplers commonly include a region of multimode waveguide that couples the optical power from the input ports to output ports. The working principle of MMI couplers depends on selfimaging effect that an interference pattern consisting of a single or multifold reproductions of the input field appears periodically when sufficient modes are excited along the MMI region [27, 28]. The propagation of higher-order modes besides the fundamental mode can couple the input power to the output ports with low insertion losses [29]. Few works based on the self-imaging have been reported in plasmonic devices, though MMI patterns have already been observed with LRSPP waveguides [2]. 
According to the principle of self-imaging effect, the beat length between two lowest-order modes of LRSPP wave can be described as

$$
L_{\pi}=\frac{\pi}{\left(\beta_{0}-\beta_{1}\right)}=\frac{\lambda}{2\left(N_{0}^{\text {eff }}-N_{1}^{\text {eff }}\right)},
$$

where $\lambda$ is the wavelength, $\beta_{0}$ and $\beta_{1}$ are propagation constants, and $N_{0}^{\text {eff }}$ and $N_{1}^{\text {eff }}$ obtained from the calculation of effective index method are effective indices of the zeroand first-order mode, respectively. Due to the symmetry of the input field at coupler entrance, only even eigenmodes can be generated when a single-mode input waveguide is centrally positioned to the MMI section. Optical reflection at the junction between the access waveguides and MMI section is ignored in the calculation.

In this paper, the permittivity of the gold stripe used is $\varepsilon=131.95+12.65 i$ at a wavelength of $1.55 \mu \mathrm{m}$. At room temperature, refractive indices of $\mathrm{Au}$ and silicon wafer are $\widetilde{n}_{\mathrm{Au}}=$ $0.55+11.5$ and $n_{\mathrm{Si}}=3.5$, respectively [8]. P(MMA-GMA) with a refractive index of 1.4801 and a thickness of $30 \mu \mathrm{m}$ is used here to accommodate LRSPP modes. Considering the attenuation induced by the resistance of metal stripe, single or multifold images of the input electromagnetic field appeared as the periodic maxima will occur periodically in accordance with the property self-imaging effect, and the length of MMI coupler is chosen to be $3000 \mu \mathrm{m}$.

2.3. Thermal Effect. It is well known that the refractive index of a material commonly is a function of its temperature and depends on thermal expansion coefficient. Polymer cladding of P(MMA-GMA) has a negative expansion coefficient, which implies that its refractive index decreases with temperature rising and follows the linear relationship

$$
n(T)=n\left(T_{0}\right)+\left(T-T_{0}\right)\left(\frac{d n}{d T}\right),
$$

where $n$ is the refractive index, $T$ is the temperature, and $d n / d T$ is referred to as the TO coefficient, which is considered to be a constant when the temperature fluctuates within a certain range. Then, the temperature generated by the applying of electrical power on the heater exhibits a maximum close to the heater and reduces gradually from it. This temperature gradient in $\mathrm{P}$ (MMA-GMA) cladding results in a negative gradient of refractive index above the metal stripe and the consequential asymmetry of refractive index breaks the condition for mode interference of LRSPP, leading to the radiation loss of the LRSPP mode. Thus, the optical output of the attenuator would be controlled by varying the temperature gradient formed in the device by electrical heating.

When a certain driving power is applied to the heater, the temperature distribution across the section is shown in Figure 2. The maximal temperature appears close to the heater and drops away from it. Here, the silicon substrate and top surface were assumed to be an efficient heat sink and exposed to still air at room temperature. The temperature dependence of the complex refractive index of gold

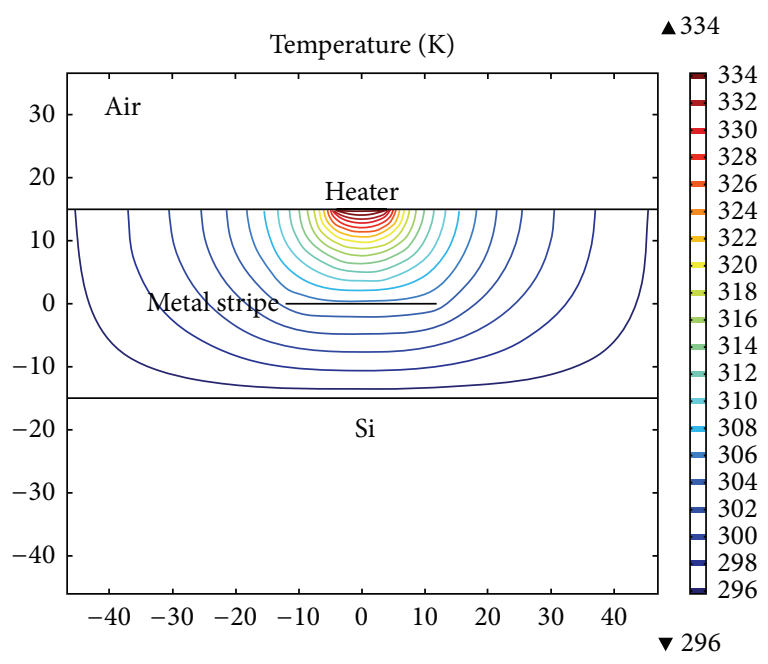

FIGURE 2: Temperature profile in the waveguide when the temperature of heater is $334 \mathrm{~K}$.

$\widetilde{n}_{\mathrm{Au}}=n+i \kappa, d n / d T=5 \times 10^{-4} / \mathrm{K}$ and $d \kappa / d T=-1.8 \times 10^{-4} / \mathrm{K}$ is used [30]. The thermal conductivity of P(MMA-GMA) used here is $0.2 \mathrm{~W} / \mathrm{m}^{\circ} \mathrm{C}$.

In essence, since the effective complex refractive index of metal depending on temperature can be evaluated from a temperature dependent Drude model $[31,32]$, the role of TO properties of metal in plasmonic structures needs to be considered when investigating the LRSPP mode change in VOA, especially when the temperature is high. The dielectric function of $\mathrm{Au}$ is dominated by free-electron contribution that the temperature increasing will lead to an increment of ohmic losses due to the electron-phonon scattering rate, which is similar to the variation of the static resistivity of metals at temperature increasing [33]. This effect benefits the application of plasmonic waveguides as optical attenuators $[34,35]$. According to the temperature distribution analysis in Figure 2, the temperature of Au stripe in the waveguide is less than $310 \mathrm{~K}$ during working. Hence, the contribution of temperature dependent dielectric coefficient of metal to the mode extinction is not so distinct as those works in [13-15], in which the metal stripe both routes the optical signal and acts as a heating element.

\section{Experiments}

3.1. Fabrication and Characterization. The optical loss of proposed LRSPP waveguide with symmetrical polymer claddings is induced by different issues, including ohmic loss of metal, scattering loss coming from the imperfect configuration of metal stripe, and intrinsic absorption loss of cladding material. All these loss-inducing factors should be restrained and minimized to eliminate excess optical loss. Here, the same polymer material of P(MMA-GMA) is used as the upper and lower cladding to guarantee the symmetry of waveguide that can reduce the propagation loss effectively. Since LRSPP waveguides with different metal stripe thicknesses and widths can provide various mode 


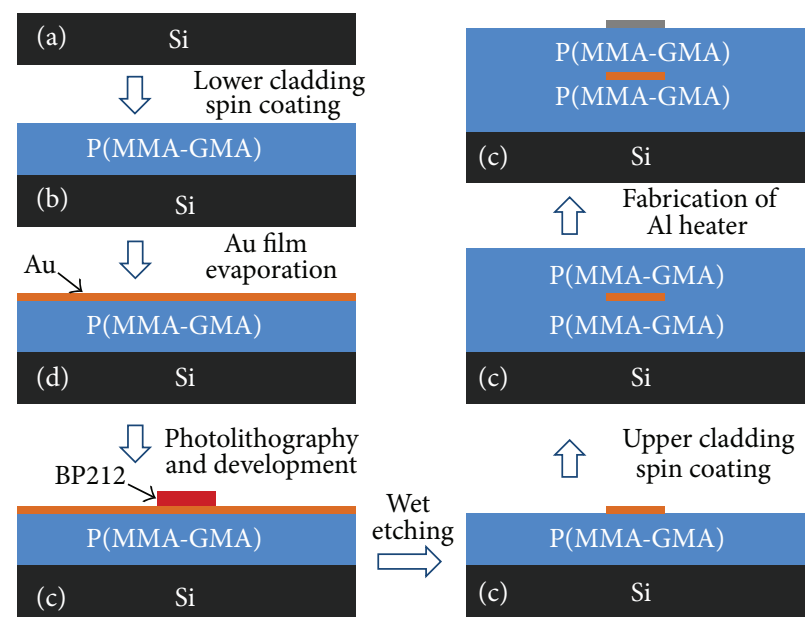

FIGURE 3: Fabrication process of the LRSPP attenuator.

field diameters, claddings are thick enough to fully support the mode field of LRSPP. The deposition techniques and parameters are crucial to obtain high quality thin metal films because discontinuous structure or variations of dielectric constant of thin film may arise from the fabrication and affect the optical characteristics of LRSPP waveguide. Thus, much experimental work has been carried out to determine the optimized technique parameters to ensure the physical quality of LRSPP waveguide, including surface roughness and configuration. Figure 3 presents the schematic diagram of fabrication process.

Firstly, a layer of $15 \mu \mathrm{m}$ thick P(MMA-GMA) was spincoated on a silicon wafer at $2500 \mathrm{rpm}$ as the lower cladding. After a subsequent bake at $120^{\circ} \mathrm{C}$ for $2.5 \mathrm{~h}$, thermal evaporation method was used to deposit high-purity (>99.999\%) solid Au thin metal film onto the polymer cladding. A calibrated quartz-crystal microbalance as the thickness monitor was used to keep the deposition at a rate of $0.05 \mathrm{~nm} / \mathrm{s}$ that is favorable to control the thickness to be about $20 \mathrm{~nm}$. To define waveguide patterns on Au film, a photoresist BP212 (Kempur Microelectronics. Inc., China) was spin-coated and patterned by ultraviolet (UV) photolithography machine (ABM Co. Inc., USA). Wet etching solution of $\mathrm{KI}: \mathrm{I}_{2}: \mathrm{H}_{2} \mathrm{O}=4: 1: 100$ was used to wipe off metal film without the protection of photoresist. After that, the sample was exposed by UV light and immersed in $1 \mathrm{wt} \% \mathrm{NaOH}$ solution and then agitated to remove the photoresist introduced in fabrication. Then, the same P(MMA-GMA) material was spin-coated and cured on the Au stripe as upper cladding. In order to avoid the damage on the metal stripe caused by stress build-up when baking the upper cladding, a step heating of $65^{\circ} \mathrm{C}$ for $20 \mathrm{~min}$, $80^{\circ} \mathrm{C}$ for $20 \mathrm{~min}$, and $90^{\circ} \mathrm{C}$ for $3 \mathrm{~h}$ was used to release inner pressure and ensure that all solvent had been evaporated from the P(MMA-GMA). Finally, a layer of $400 \mathrm{~nm}$ Al film was thermally evaporated and patterned with photolithography. Wet etching was adopted to form a heater on the upper cladding. Before the measurement, the waveguide sample was sliced by a wafer dicing machine DAD-3220 (DISCO Co. Inc., Japan) to minimize the uncertainty of input/output coupling loss.
AFM images were recorded with a multimode scanning probe microscope CSPM5000 (Being Nano-Instrument Ltd., China) that operated in contact mode to examine the detailed morphology of an $8 \mu \mathrm{m}$ wide Au stripe on a $20 \times 20 \mu \mathrm{m}^{2}$ area, as shown in Figure 4 . The surface scan exhibits a thickness of about $20 \mathrm{~nm}$ with a smooth surface, as shown in Figure 4(a). No obvious evaporation spits or metal wing along the edge of stripe is observed, which proves the effectiveness of photolithography and etching. Steep side wall shown in Figure 4(b) provides a good mode field control that will be discussed in Section 4.

3.2. Measurement. The schematic diagram of transmission characteristics measurement setup of LRSPP attenuator is shown in Figure 5. The fiber tips need to be cleaned and checked to ensure that no damage is on their facets. The end-fire excitation of LRSPP mode guiding at telecommunication wavelengths along thin Au stripes with finite width embedded in dielectric has been experimentally proved [7]. Then, the light at $1550 \mathrm{~nm}$ from a tunable laser source TSL210 (Santec Co., Japan) was perpendicularly polarized to the waveguide plane by passing through a polarization controller and then launched into the waveguide to excite LRSPP mode. A standard SM fiber was used to couple the output signal to an optical power meter or the photoreceiver. The output fiber alignment was optimized with a five-axis position control to maximize the optical output of LRSPP mode, so did the input fiber alignment, until the best condition was achieved, as shown in Figure 5(a). The far-field output of $\mathrm{Au}$ stripe waveguide was monitored by an infrared (IR) camera through 200 magnifications, as shown in Figure 5(b). To study the dynamic performance, the attenuator was fixed on a thermoelectric controller, temperature of which can be maintained at a constant value of about $25^{\circ} \mathrm{C}$. The electrical current was introduced to the heater with microprobes. The time response of VOA was observed and recorded through an oscillograph. 


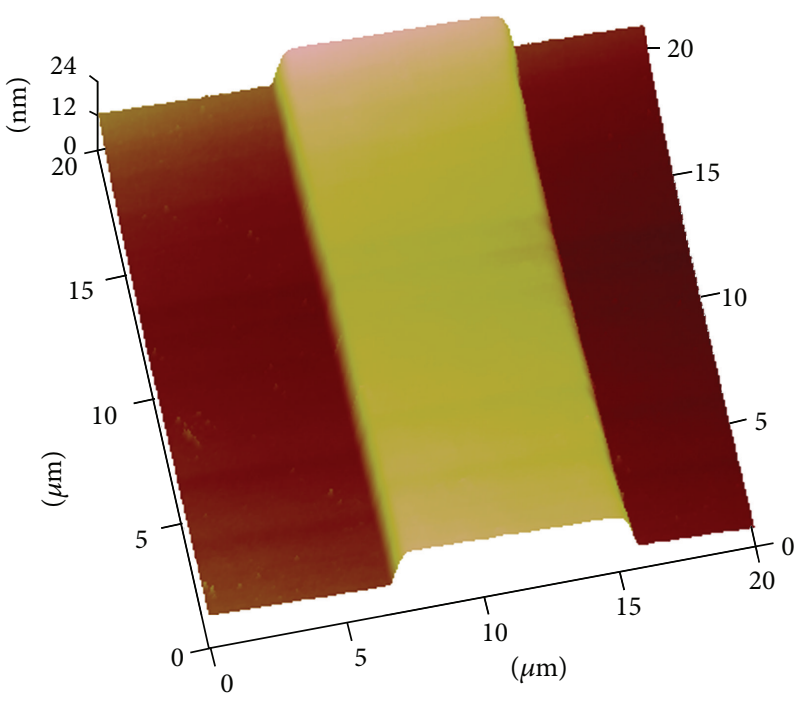

(a)

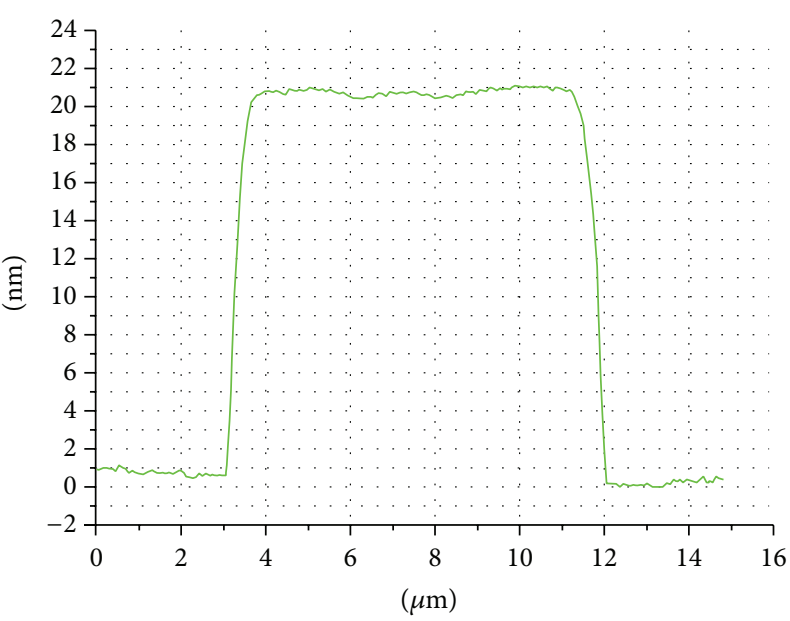

(b)

FIgURE 4: AFM images of the evaporated Au stripe on P(MMA-GMA) lower cladding (a) three-dimensional configuration of Au stripe and (b) line scan across the Au stripe.

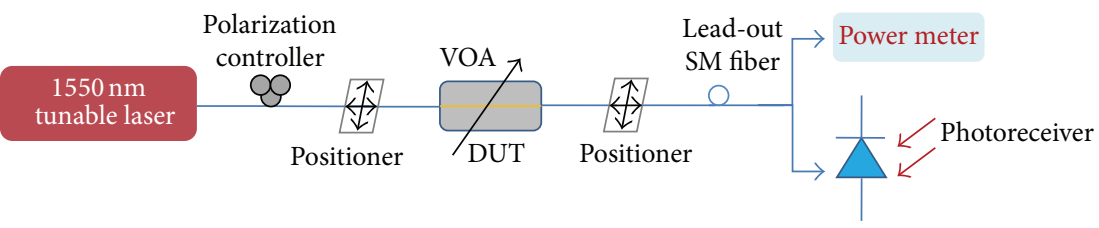

(a)

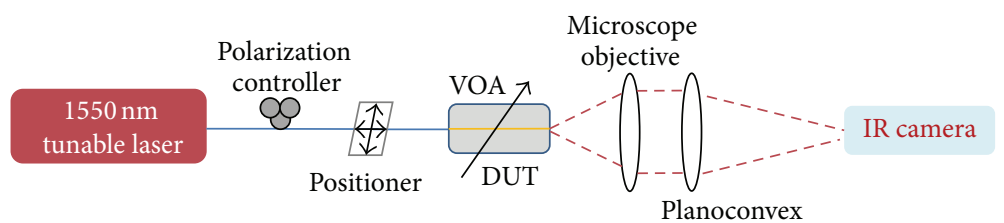

(b)

FIGURE 5: Schematic setup for measuring (a) insertion loss and time response and (b) far-field output pattern of VOA.

\section{Results and Discussion}

4.1. Refractive Index of Polymer Cladding. Comparing with higher index cladding materials, polymer with lower refractive index can decrease the propagation loss of LRSPP due to its less mode binding. As an optical signal transmission medium, P(MMA-GMA) material synthesized by ourselves is used in this study. Its number average molecular weight $M_{n}$ and weight average molecular weight $M_{w}$ are 64547 and 413466, respectively. By cut-back method, the propagation loss of a fabricated rectangular P(MMA-GMA) waveguide is less than $3 \mathrm{~dB} / \mathrm{cm}$ [36]. According to the analysis in Section 3, refractive index matching of the upper and lower cladding layers is critically required due to the symmetrical condition of LRSPP waveguide. Or else, the optical mode will radiate, resulting in a large attenuation [2, 37]. Moreover, the mode field of LRSPP is designed to accommodate that of a single mode optical fiber which expands to nine micrometers or even larger; thus the refractive index of cladding should be well controlled surrounding the Au stripe. However, the P(MMA-GMA) lower cladding experienced a second thermal curing in photolithography process and the upper cladding formation, which may change its refractive index. Therefore, the optical stability of P(MMA-GMA) should be confirmed experimentally. Figure 6 shows the refractive index of polymer cladding as a function of curing time measured by an M-2000UI ellipsometer (J. A. Woollam CO., Inc.). Typical refractive index of the film is 1.4802 for TM polarization at $1550 \mathrm{~nm}$ after $3 \mathrm{~h}$ baking. Considering the uncertainty of the measurement, no refractive index change larger than $3 \times 10^{-4}$ is observed with $8 \mathrm{~h}$ baking at $120^{\circ} \mathrm{C}$, which proves the thermal stability of cladding. This result is the average of 3 different samples and convinced to be within the uncertainty of measurement. Thus, thermal curing material P(MMA-GMA) and the fabrication process can guarantee equal refractive indices for both claddings. 


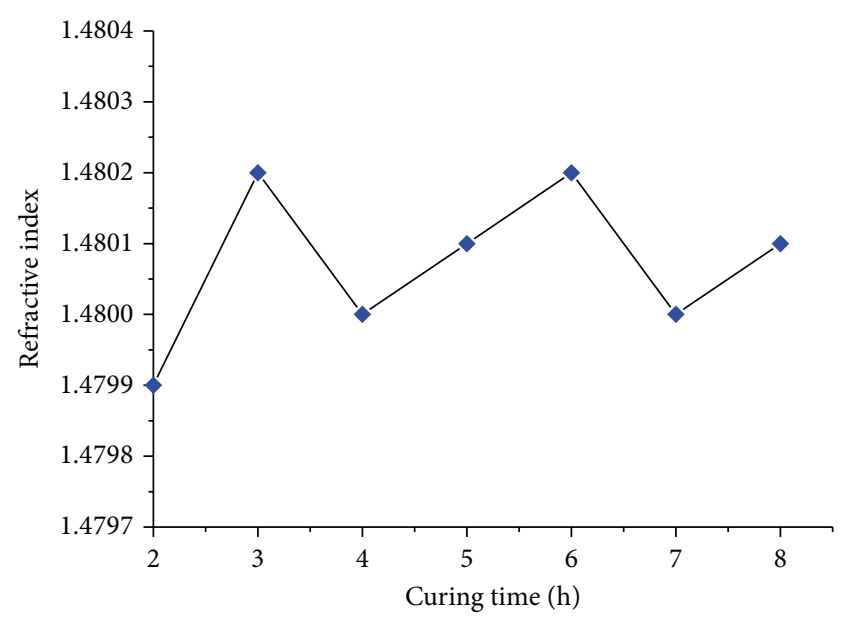

FIGURE 6: Experimental P(MMA-GMA) refractive index change with the curing time.

4.2. Insertion Loss. When the heater is working, the optical power at the output port is monitored with the infrared camera or the optical power meter aligned with IR camera removed to determine the optical extinction ratio of VOA. The optical attenuation of VOA based on LRSPP waveguide can be described as the light expelled from plasmonic waveguide with reducing the RI around Au stripe. Because of the negative thermooptic coefficient of P(MMA-GMA) cladding, the LRSPP mode will be cut off when the electrical power is applied on the heater. The intensity distribution of the fundamental LRSPP mode at $1.55 \mu \mathrm{m}$ is shown in Figure 7. Because of the distinguish index difference between the gold and polymer cladding, no significant mode mismatch induced loss is observed. With the applied electrical power increasing, the optical output changes from nonradiative mode in Figure 7(a) to radiative mode in Figures 7(b)-7(e) and finally extinguished in Figure $7(\mathrm{f})$. As shown in Figures $7(\mathrm{~b})-7(\mathrm{e})$, the optical power radiates in all directions from the Au stripe to polymer claddings.

The optical output power as a function of the driving power is described in Figure 8. The extinction ratio of attenuator is about $12 \mathrm{~dB}$ at a driving power of $69 \mathrm{~mW}$. Compared with other VOAs based on TO effect, it can be seen that, for a $3 \mathrm{~mm}$ long LRSPP based in-line extinction modulator, a power consumption of about $48 \mathrm{~mW}$ is needed to achieve the same optical attenuation, which is comparable with that of this work [10]. For polymeric waveguide VOAs, the power consumptions are both about $17 \mathrm{~mW}$, which is lower than that in this paper due to the large TO coefficient of polymers $[38,39]$. This relatively high power consumption is mainly due to the thick polymer cladding that extends the distance of thermal diffusion. To establish the same temperature difference between the upside and underside of $\mathrm{Au}$ stripe, more power is needed.

As shown in Figure 8, the measured optical signal attenuation is not so remarkable, which is attributed to the following reasons. Firstly, the optical field extending to surrounded polymer claddings leads to a large mode size, though the LRSPP modes are controlled on the metal surface. The optical modulation induced by effective refractive index changing is unable to block all optical signal propagation. Secondly, the design of heater is supposed to be optimized. There exist different manners of heater placement on the MMI waveguide, and different heater dimensions will lead to various optical attenuations. If the heater design in this paper was further optimized, we believe the attenuation characteristic can be improved. In fact, the process induced fabrication errors, such as scattering caused by surface roughness of Au stripe, is also an origin of low attenuation. Thus, a moderate optical attenuation is obtained in this work.

The insertion loss variation as driving power is over $70 \mathrm{~mW}$ can be explained that some higher-order modes are excited as the LRSPP mode propagates from the input single mode waveguide to the MMI coupler, which is due to the limited length of tapered region. This may result in multimode interference in the MMI waveguide region. Therefore, the insertion loss varies sinuously as the driving power is larger than $70 \mathrm{~mW}$, which weakens the mode reflection and leading to the restoration of output power.

4.3. Time Response. The response times are measured by an oscilloscope through collecting the output optical power with a butt-coupled single mode fiber, which is connected to an optical power meter. A $100 \mathrm{~Hz}$ square waveform drive current is applied on the heater. Since the rising edge of drive current induces mode cutoff, the thermal rise time which follows the rising edge of the drive current corresponds to the optical fall time, and vice versa. When the attenuator is subjected to a square waveform drive current, the rise and fall times are defined as the time that the detected optical power increases from 10 to $90 \%$ or decreases from 90 to $10 \%$ of its peak value, respectively.

Figure 9 shows the time response of VOA. The measured optical rise time and fall time are 0.57 and $0.87 \mathrm{~ms}$, respectively. The fall time is much longer than the rise time, which is usually observed in thermooptic attenuators. This can be explained that the temperature change in upper cladding that impacts the optical characteristic of LRSPP mode is fast due to the relatively short diffusion path existing between the heater and Au stripes when injecting the driving current, while the optical rise time is decided by thermal fall time which corresponds to the cooling process that restores the refractive index symmetry of polymer claddings after turning off the drive current. Because the heat accumulated in $30 \mu \mathrm{m}$ thick claddings must diffuse from the optical region to the heat sink of silicon substrate with little heat evacuating through the air, this process is slower than the construction of temperature asymmetry in polymer claddings. Thus, the thermal rise time is faster than the optical fall time. However, the speed of TO effect can be increased remarkably when $\mathrm{P}$ (MMA-GMA) with a low thermal conductivity of $0.2 \mathrm{~W} / \mathrm{m}^{\circ} \mathrm{C}$ is replaced by materials 


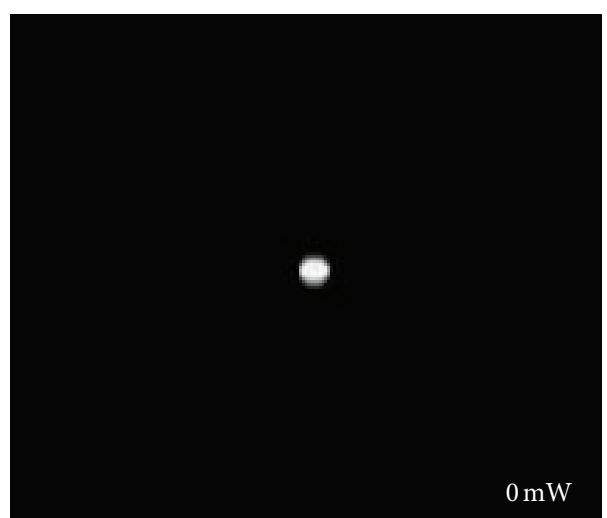

(a)

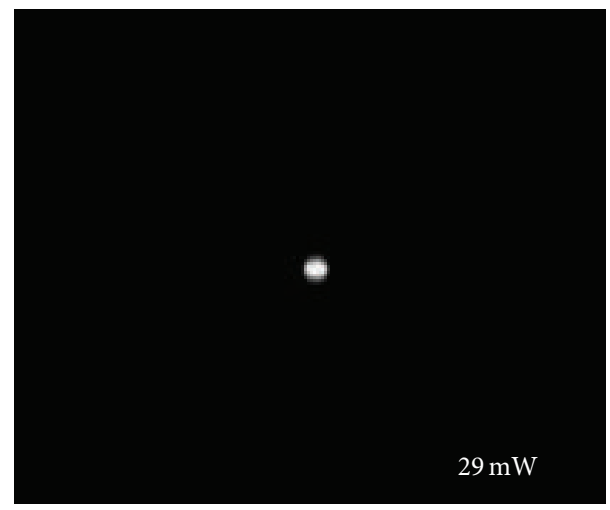

(c)

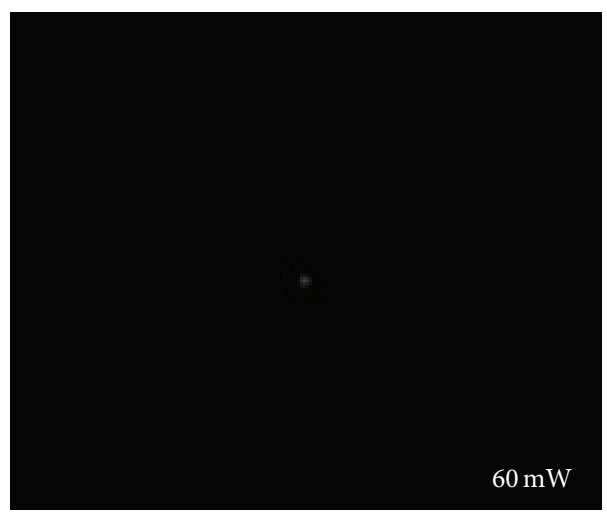

(e)

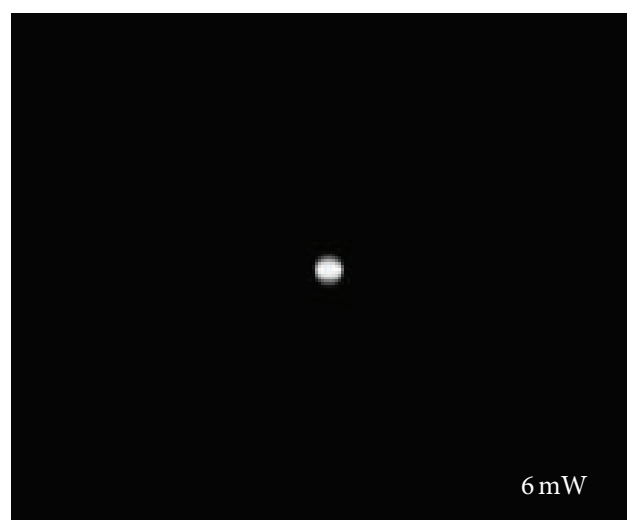

(b)

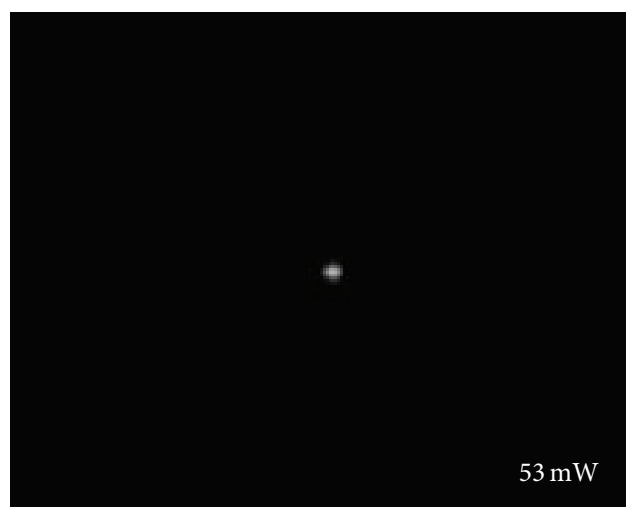

(d)

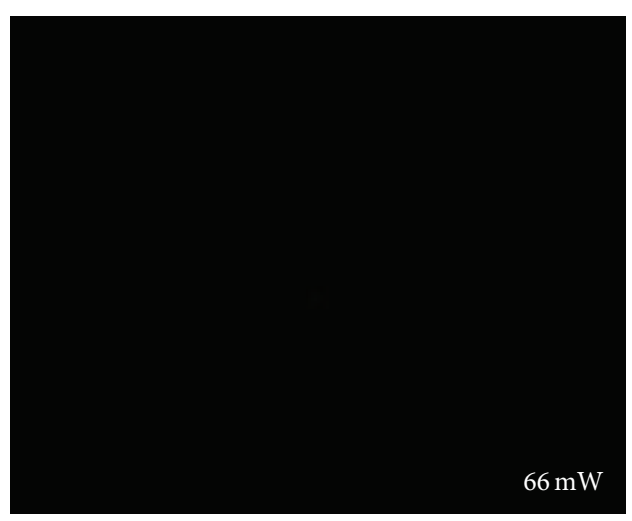

(f)

FIGURE 7: The intensity distribution of fundamental LRSPP mode at $1.55 \mu \mathrm{m}$ captured by IR camera with different electrical driving power of (a) $0 \mathrm{~mW}$, (b) $6 \mathrm{~mW}$, (c) $29 \mathrm{~mW}$, (d) $53 \mathrm{~mW}$, (e) $60 \mathrm{~mW}$, and (f) $66 \mathrm{~mW}$ applied on the heater.

with higher thermal conductivity. Thus, it is convincing that the time response of this kind of VOA can be significantly improved and has a promising future for the application in optical communication systems.

\section{Conclusions}

A thermooptic attenuator based on LRSPP waveguide was investigated. Our investigations of morphological and structural characteristics of Au stripe after etching show that no obvious evaporation spits or metal wing exists along the edge of stripe and the steep side wall provides good mode field control proved by IR photos of field patterns. The refractive index symmetry of waveguide is guaranteed by polymer cladding of P(MMA-GMA). Variable optical attenuation is realized by the thermooptic effect induced LRSPP mode radiation at telecommunication wavelength. The fabrication technology based on true planar processing and favorable performance suggests potential integration with other photonic devices. 


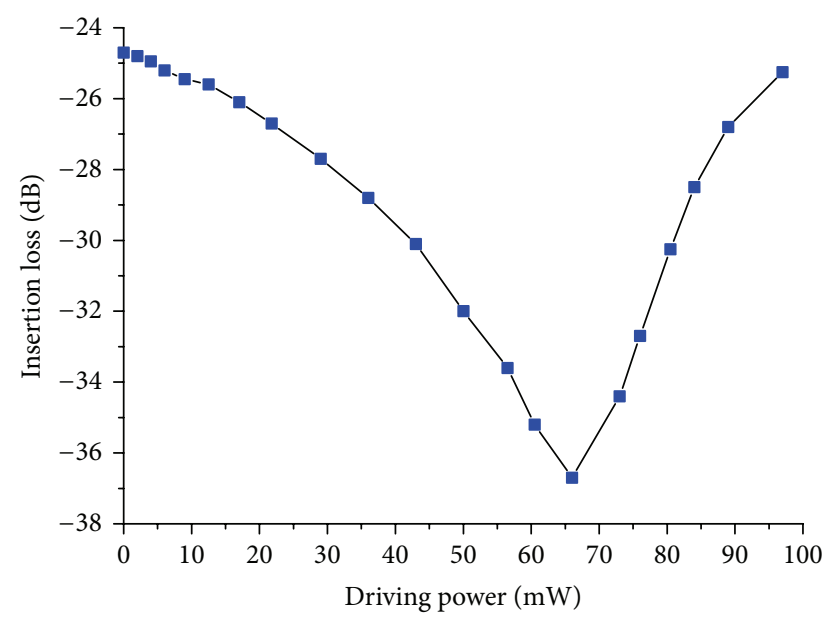

Figure 8: Experimental attenuation characteristic versus electrical power.

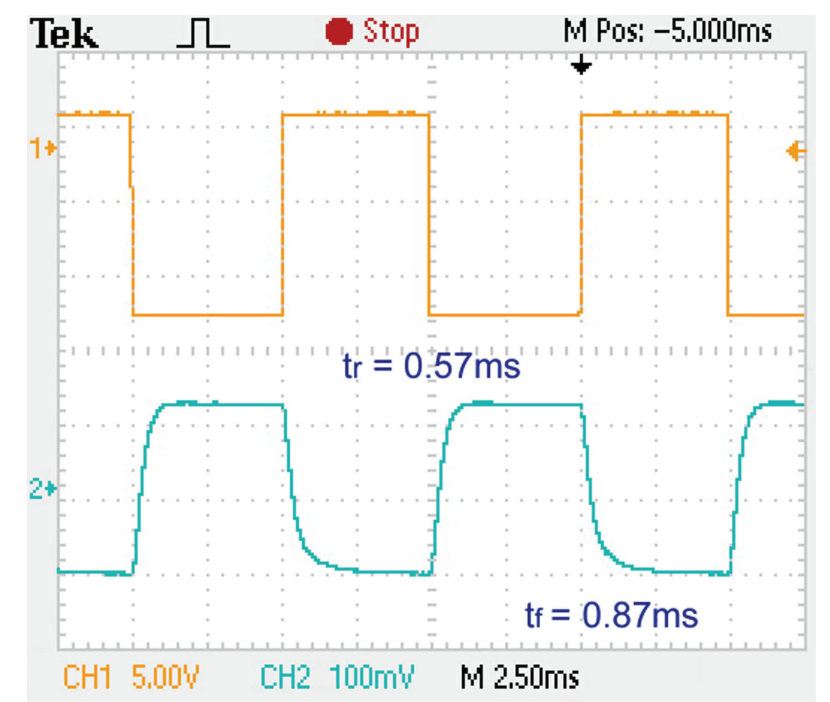

FIGURE 9: The response time of VOA is measured to be $0.57 \mathrm{~ms}$ for heating and $0.87 \mathrm{~ms}$ for cooling at $100 \mathrm{~Hz}$ for a fixed driving power.

\section{Conflict of Interests}

The authors declare that there is no conflict of interests regarding the publication of this paper.

\section{Acknowledgments}

This work is supported by the National Natural Science Foundation of China (nos. 61177027, 61107019, 61205032, and 61261130586), Program for Special Funds of Basic Science \& Technology of Jilin University (nos. 201100253 and 201103071), and China Postdoctoral Science Foundation (no. 2012M510900).

\section{References}

[1] T. Nikolajsen, K. Leosson, I. Salakhutdinov, and S. I. Bozhevolnyi, "Polymer-based surface-plasmon-polariton stripe waveguides at telecommunication wavelengths," Applied Physics Letters, vol. 82, no. 5, pp. 668-670, 2003.

[2] A. Boltasseva, T. Nikolajsen, K. Leosson, K. Kjaer, M. S. Larsen, and S. I. Bozhevolnyi, "Integrated optical components utilizing long-range surface plasmon polaritons," Journal of Lightwave Technology, vol. 23, no. 1, pp. 413-422, 2005.

[3] J. Takahara and M. Miyata, "Mutual mode control of short- and long-range surface plasmons," Optics Express, vol. 21, no. 22, pp. 27402-27410, 2013.

[4] J. Lee and M. A. Belkin, "Widely tunable thermo-optic plasmonic bandpass filter," Applied Physics Letters, vol. 103, no. 18, Article ID 181115, 2013.

[5] A. Khan, O. Krupin, E. Lisicka-Skrzek, and P. Berini, "MachZehnder refractometric sensor using long-range surface plasmon waveguides," Applied Physics Letters, vol. 103, no. 11, Article ID 111108, 2013.

[6] T. Zhang, G. Qian, Y.-Y. Wang et al., "Integrated optical gyroscope using active Long-range surface plasmon-polariton waveguide resonator," Scientific Reports, vol. 4, article 3855, 2014.

[7] R. Charbonneau, P. Berini, E. Berolo, and E. Lisicka-Shrzek, "Experimental observation of plasmon-polariton waves supported by a thin metal film of finite width," Optics Letters, vol. 25, no. 11, pp. 844-846, 2000.

[8] R. Charbonneau, C. Scales, I. Breukelaar et al., "Passive integrated optics elements based on long-range surface plasmon polaritons," Journal of Lightwave Technology, vol. 24, no. 1, pp. 477-494, 2006.

[9] Y.-J. Tsai, A. Degiron, N. M. Jokerst, and D. R. Smith, "Plasmonic Multi-Mode Interference Couplers," Optics Express, vol. 17, no. 20, pp. 17471-17482, 2009.

[10] T. Nikolajsen, K. Leosson, and S. I. Bozhevolnyi, "In-line extinction modulator based on long-range surface plasmon polaritons," Optics Communications, vol. 244, no. 1-6, pp. 455459, 2005.

[11] T. Nikolajsen, K. Leosson, and S. I. Bozhevolnyi, "Surface plasmon polariton based modulators and switches operating at telecom wavelengths," Applied Physics Letters, vol. 85, no. 24, pp. 5833-5835, 2004.

[12] G. Gagnon, Thermo-optic variable optical attenuators using plasmon polariton waveguides [M.S. thesis], University of Ottawa, Ottawa, Canada, 2004.

[13] G. Gagnon, N. Lahoud, G. A. Mattiussi, and P. Berini, “Thermally activated variable attenuation of long-range surface plasmon-polariton waves," Journal of Lightwave Technology, vol. 24, no. 11, pp. 4391-4402, 2006.

[14] C. Chiu, E. Lisicka-Skrzek, R. N. Tait, and P. Berini, "Fabrication of surface plasmon waveguides and devices in Cytop with integrated microfluidic channels," Journal of Vacuum Science and Technology B, vol. 28, no. 4, pp. 729-735, 2010.

[15] H. Fan, R. Buckley, and P. Berini, "Passive long-range surface plasmon-polariton devices in Cytop," Applied Optics, vol. 51, no. 10, pp. 1459-1467, 2012.

[16] J. Gosciniak and S. I. Bozhevolnyi, "Performance of thermooptic components based on dielectric-loaded surface plasmon polariton waveguides," Scientific Reports, vol. 3, article 1803, 2013. 
[17] S. Talukder, P. Kumar, and R. Pratap, "Electric current-Induced mass flow in very thin infinite metallic films," IEEE Transactions on Electron Devices, vol. 60, no. 9, pp. 2877-2883, 2013.

[18] S. Kaya, J.-C. Weeber, F. Zacharatos et al., "Photo-thermal modulation of surface plasmon polariton propagation at telecommunication wavelengths," Optics Express, vol. 21, no. 19, pp. 22269-22284, 2013.

[19] K. Leosson, T. Rosenzveig, P. G. Hermannsson, and A. Boltasseva, "Compact plasmonic variable optical attenuator," Optics Express, vol. 16, no. 20, pp. 15546-15552, 2008.

[20] Q. Zhao, K. Cui, X. Feng, F. Liu, W. Zhang, and Y. Huang, "Variable optical attenuator based on photonic crystal waveguide with low-group-index tapers," Applied Optics, vol. 52, no. 25, pp. 6245-6249, 2013.

[21] Y.-J. He, F. Li, and Y.-L. Liu, "Silicon-on-insulator based electrooptic variable optical attenuator with a series structure," Chinese Physics Letters, vol. 22, no. 1, pp. 95-98, 2005.

[22] S. Park, T. Tsuchizawa, T. Watanabe et al., "Monolithic integration and synchronous operation of germanium photodetectors and silicon variable optical attenuators," Optics Express, vol. 18, no. 8, pp. 8412-8421, 2010.

[23] J. Lee, F. Lu, and M. A. Belkin, "Widely-tunable optical bandpass filter based on long-range surface plasmon polaritons," in Plasmonics: Metallic Nanostructures and Their Optical Properties X, vol. 8457 of Proceedings of SPIE, 2012.

[24] D. Kalavrouziotis, S. Papaioannou, G. Giannoulis et al., " $0.48 \mathrm{~Tb} / \mathrm{s}(12 \times 40 \mathrm{~Gb} / \mathrm{s})$ WDM transmission and high-quality thermo-optic switching in dielectricloaded plasmonics," Optics Express, vol. 20, no. 7, pp. 7655-7662, 2012.

[25] J. Jiang, C. L. Callender, S. Jacob et al., "Long-range surface plasmon polariton waveguides embedded in fluorinated polymer," Applied Optics, vol. 47, no. 21, pp. 3892-3900, 2008.

[26] Y. O. Noh, M.-S. Yang, Y. H. Won, and W.-Y. Hwang, "PLC-type variable optical attenuator operated at low electrical power," Electronics Letters, vol. 36, no. 24, pp. 2032-2033, 2000.

[27] O. Bryngdahl, "Image formation using self-imaging techniques," Journal of the Optical Society of America, vol. 63, no. 4, pp. 416-419, 1973.

[28] R. Ulrich, "Image formation by phase coincidences in optical waveguides," Optics Communications, vol. 13, no. 3, pp. 259-264, 1975.

[29] L. B. Soldano and E. C. M. Pennings, "Optical multi-mode interference devices based on self-imaging: principles and applications," Journal of Lightwave Technology, vol. 13, no. 4, pp. 615-627, 1995.

[30] M. Rashidi-Huyeh and B. Palpant, "Counterintuitive thermooptical response of metal-dielectric nanocomposite materials as a result of local electromagnetic field enhancement," Physical Review B, vol. 74, no. 7, Article ID 075405, 2006.

[31] S. K. Özdemir and G. Turhan-Sayan, "Temperature effects on surface plasmon resonance: design considerations for an optical temperature sensor," Journal of Lightwave Technology, vol. 21, no. 3, pp. 805-814, 2003.

[32] C. S. Moreira, A. M. N. Lima, H. Neff, and C. Thirstrup, "Temperature-dependent sensitivity of surface plasmon resonance sensors at the gold-water interface," Sensors and Actuators $B$, vol. 134, no. 2, pp. 854-862, 2008.

[33] X.-Y. Zhang, T. Zhang, A.-M. Hu, X.-J. Xue, P.-Q. Wu, and Q.-Y. Chen, "Tunable microring resonator based on dielectric-loaded surface plasmon polariton waveguides," Journal of Nanoscience and Nanotechnology, vol. 11, no. 12, pp. 10520-10524, 2011.
[34] K. Leosson, T. Nikolajsen, A. Boltasseva, and S. I. Bozhevolnyi, "Long-range surface plasmon polariton nanowire waveguides for device applications," Optics Express, vol. 14, no. 1, pp. 314319, 2006.

[35] X.-Y. Zhang, A. Hu, T. Zhang, X.-J. Xue, J. Z. Wen, and W. W. Duley, "Subwavelength plasmonic waveguides based on $\mathrm{ZnO}$ nanowires and nanotubes: a theoretical study of thermo-optical properties," Applied Physics Letters, vol. 96, no. 4, Article ID 043109, 2010.

[36] X. Fei, N. Fu, Y. Wang et al., "Synthesis and characterization of crosslinkable Poly(MMA-co-GMA) and its application in arrayed waveguide grating," Chemical Journal of Chinese Universities, vol. 27, no. 3, pp. 571-574, 2006.

[37] P. Berini, "Long-range surface plasmon-polariton waveguides in silica," Journal of Applied Physics, vol. 102, no. 5, Article ID 053105, 2007.

[38] Y.-O. Noh, C.-H. Lee, J.-M. Kim et al., "Polymer waveguide variable optical attenuator and its reliability," Optics Communications, vol. 242, no. 4-6, pp. 533-540, 2004.

[39] M.-C. Oh, S.-H. Cho, Y.-O. Noh, H.-J. Lee, J.-J. Joo, and M.H. Lee, "Variable optical attenuator based on large-core singlemode polymer waveguide," IEEE Photonics Technology Letters, vol. 17, no. 9, pp. 1890-1892, 2005. 

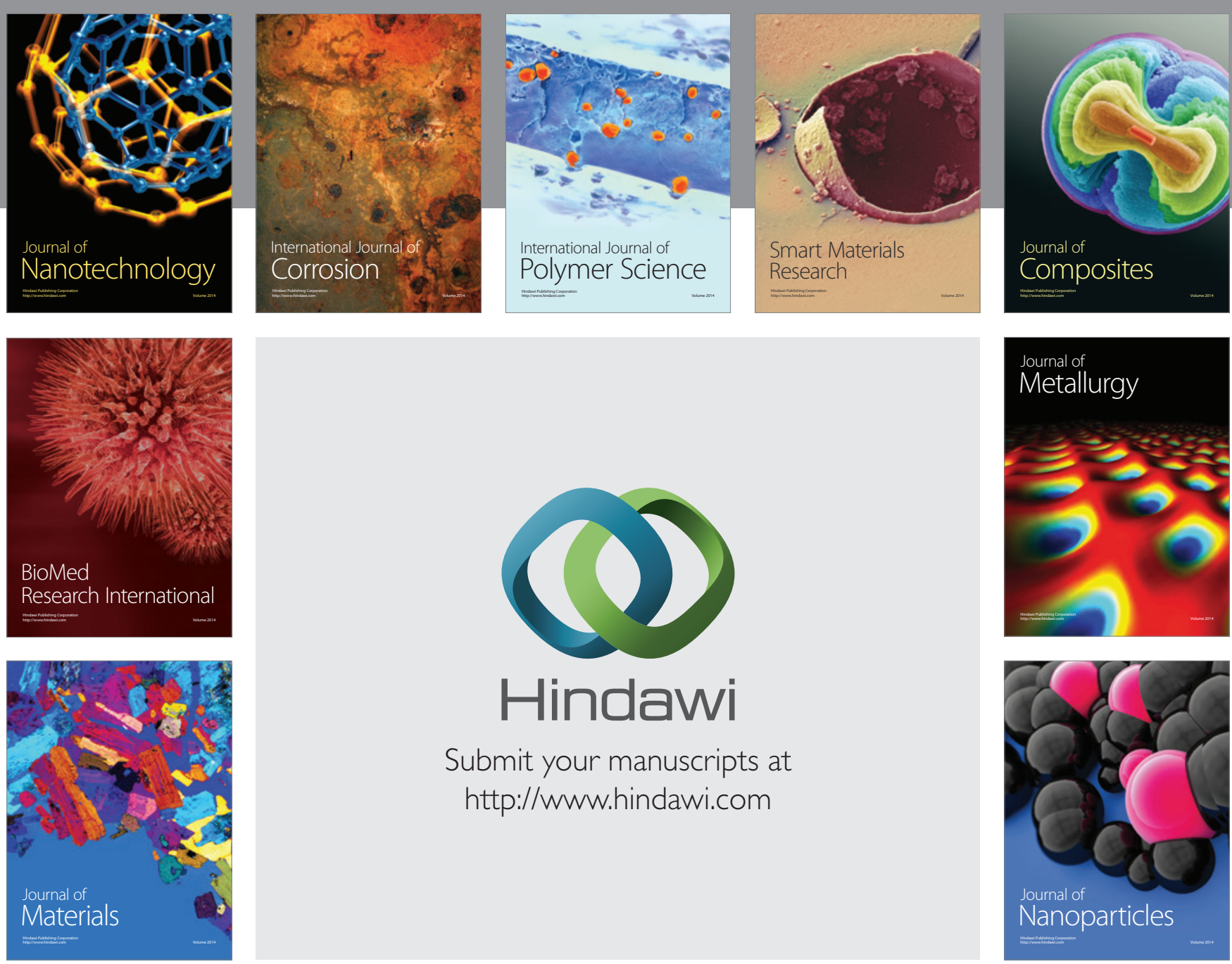

Submit your manuscripts at http://www.hindawi.com
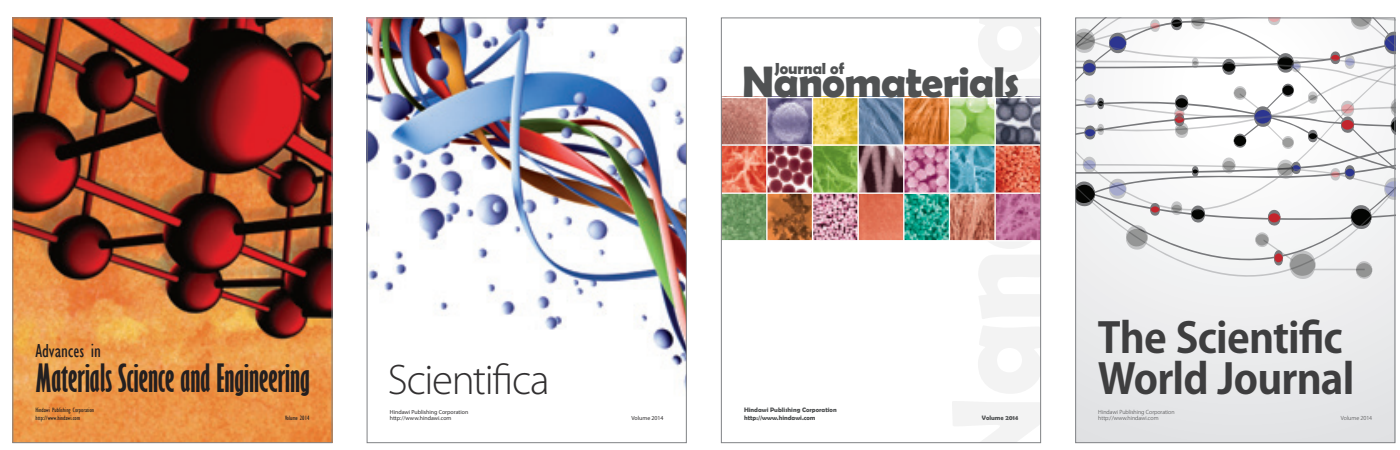

\section{The Scientific World Journal}
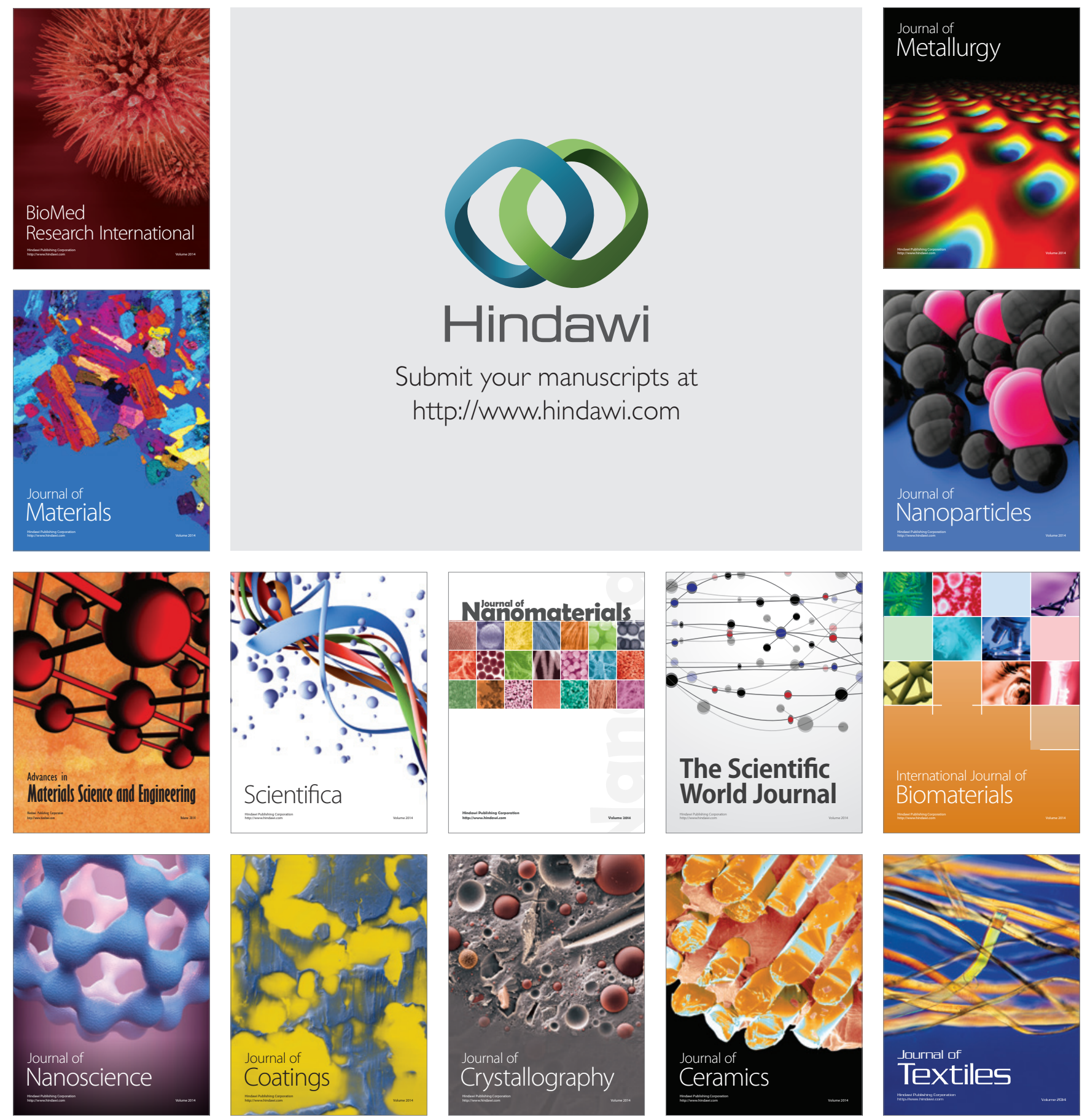